\title{
ON THE ASYMPTOTIC PARTITION OF ENERGY IN LINEAR THERMOELASTICITY*
}

\author{
BY \\ S. CHIRIŢĂ \\ University of Iaşi, România
}

1. Introduction. The question of partition of energy in the asymptotic form was first investigated by Lax and Phillips [1] and Brodsky [2]. Further, this problem has been studied by Goldstein [3,4]. In his elegant analysis of the abstract wave equation, Goldstein applies the semigroup theory in order to obtain an equipartition theorem stating that the difference of the kinetic energy and the potential energy vanishes as the time approaches infinity. By means of the Paley-Wiener theorem, Duffin [5] has shown that if a solution of the classical wave equation has compact support, then after a finite time the kinetic energy of the wave is constant and equals the potential energy.

Levine [6] later treated an abstract version of Goldstein's approach by use of the Lagrange identity method. His result represents a simplified proof that asymptotic equipartition occurs between the Cesàro means of the kinetic and potential energies, a fact first demonstrated by Goldstein [4]. The asymptotic equipartition between the mean kinetic and strain energies within the context of linear elastodynamics was established by Day [7].

In this paper we consider the classical linear theory of thermoelasticity for inhomogeneous and anisotropic materials. We investigate the asymptotic partition of total energy for the solutions of the initial boundary value problems of linear thermoelasticity. In fact, we materialize the idea of partition in terms of the asymptotic behavior in the Cesàro sense of various parts of the total energy associated to a solution. Thus we prove, among other things, that the mean thermal energy tends to zero as time goes to infinity. We demonstrate that the asymptotic equipartition occurs between the Cesàro means of the kinetic and strain energies. This proves that thermal effects do not influence explicitly the asymptotic equipartition of the mean kinetic and strain energies. Therefore, the result established by Day in [7] for linear elastodynamics continues to hold in the framework of dynamic linear thermoelasticity.

\footnotetext{
${ }^{*}$ Received May 7, 1986.
} 
The system in question consists of the hyperbolic equations of motion coupled with the parabolic equation of energy and, therefore, does not belong to one of the categories considered in previous papers. Of course, it is possible to include in our treatment suitably abstract versions (see, for example, the approach devised by Levine in [8]), but since this generalization is somewhat standard, we prefer instead to emphasize the technique itself in the classical linear theory of thermoelasticity.

Our method of proof uses Levine's refinement of the Lagrange-Brun identities. This method is a direct one and it does not rely on any special features of the thermoelastic continuum such as the homogeneity and isotropy of the material.

Finally, we remark that for isotropic and homogeneous linear thermoelasticity, the partition of energy for transverse and longitudinal waves was studied by Dassios and Grillakis [9]. Thus, for transverse waves it is shown that the total energy is conserved and, in finite time, is divided into equal parts of kinetic and strain energies. For longitudinal waves it is established that the total energy is divided equally between the strain energy and a convex combination (depending only on the coupling parameter) of the kinetic and thermal energies after a large lapse of time. The results are established by means of Fourier transform and asymptotic analysis.

2. Preliminaries. Throughout this paper we shall consider a thermoelastic solid occupying in the reference configuration the properly regular region $B$ of Euclidean three-dimensional space $E^{3}$. We assume that $B$ is bounded by the piecewise smooth surface $\partial B$ [10]. The motion of the body is referred to the reference configuration and a fixed set of rectangular Cartesian axes, relative to which $B$ is at rest to the uniform temperature $\theta_{0}>0$.

For a material point with configuration $\mathbf{x}$ at time $t$, let $\mathbf{u}(\mathbf{x}, t)$ and $\theta(\mathbf{x}, t)$ be the displacement and temperature deviation, respectively, from the natural state of the reference configuration. By $\rho(\mathbf{x})$ we denote the density at the point $\mathbf{x}$ of the natural state. A superposed dot denotes the derivative with respect to the time variable and $\nabla$ denotes the gradient operator. We denote by $\mathbf{n}$ the outward unit normal, by $d V$ the element of volume, and by $d A$ the element of surface area.

In the absence of the body force and heat supply fields, the fundamental system of field equations for the linear theory of thermoelasticity [11] consists of the equation of motion

$$
\rho \ddot{\mathbf{u}}=\operatorname{div} \mathbf{S},
$$

the energy equation

$$
c \dot{\theta}=\theta_{0} \mathbf{M} \cdot \dot{\mathbf{E}}-\operatorname{div} \mathbf{q},
$$

the stress-strain-temperature relation

$$
\mathbf{S}=\mathbf{C}[\mathbf{E}]+\theta \mathbf{M}
$$

the heat conduction equation

$$
\mathbf{q}=-\mathbf{K} \nabla \boldsymbol{\theta}
$$

and the strain-displacement relation

$$
\mathbf{E}=\operatorname{sym} \nabla \mathbf{u} .
$$


Here $\mathbf{E}, \mathbf{S}$, and $\mathbf{q}$ are the strain, stress, and heat flux, respectively; while $\mathbf{C}, \mathbf{M}, \mathbf{K}$, and $c$ are the elasticity, stress-temperature, conductivity, and specific heat fields, respectively. The fourth-order tensor $\mathbf{C}$ is symmetric; that is, for any pair of symmetric (second-order) tensors $\mathbf{A}$ and $\mathbf{B}$

$$
\mathbf{A} \cdot \mathbf{C}[\mathbf{B}]=\mathbf{B} \cdot \mathbf{C}[\mathbf{A}] \text {. }
$$

The stress-temperature tensor is symmetric,

$$
\mathbf{M}=\mathbf{M}^{T} .
$$

Furthermore, we make the common assumption that the conductivity tensor $\mathbf{K}$ is symmetric, i.e.,

$$
\mathbf{K}=\mathbf{K}^{T}
$$

A further consequence of the heat conduction inequality is the following dissipation inequality:

$$
\nabla \boldsymbol{\theta} \cdot \mathbf{K} \nabla \boldsymbol{\theta} \geqslant 0 \text {. }
$$

In what follows we assume that the elasticity tensor $\mathbf{C}$, the stress-temperature tensor $\mathbf{M}$, the conductivity tensor $\mathbf{K}$, the specific heat $c$, and the density $\rho$ are prescribed fields, and that $\mathbf{C}, \mathbf{M}$, and $\mathbf{K}$ are smooth on $\bar{B}$ while $c$ and $\rho$ are continuous on $\bar{B}$.

Introducing $\mathbf{S}$ and $\mathbf{q}$ from (3) and (4) into (1) and (2) and using the relations (5), (6), and (7), we obtain the coupled system of linear thermoelastic equations

$$
\begin{gathered}
\rho \ddot{\mathbf{u}}=\operatorname{div}(\mathbf{C}[\nabla \mathbf{u}]+\theta \mathbf{M}), \\
c \dot{\boldsymbol{\theta}}=\theta_{0} \mathbf{M} \cdot \nabla \dot{\mathbf{u}}+\operatorname{div}(\mathbf{K} \nabla \boldsymbol{\theta}),
\end{gathered}
$$

in $B \times(0, \infty)$.

Let $\partial B_{1}, \partial B_{2}, \partial B_{3}$, and $\partial B_{4}$ be fixed subsets of $\partial B$ such that $\overline{\partial B_{1}} \cup \partial B_{2}=\overline{\partial B_{3}} \cup \partial B_{4}=$ $\partial B$ and $\partial B_{1} \cap \partial B_{2}=\partial B_{3} \cap \partial B_{4}=\varnothing$. We assume that a scalar field $Q>0$ is assigned on $\partial B_{4}$.

By a solution of the mixed initial boundary value problem in $B \times(0, \infty)$ we mean a pair $(\mathbf{u}, \theta)$ satisfying Eqs. (10) and (11) for all $(\mathbf{x}, t) \in B \times(0, \infty)$, together with boundary conditions

$$
\begin{array}{ccc}
\mathbf{u}=\mathbf{0} \text { on } \quad \partial B_{1} \times(0, \infty), & (\mathbf{C}[\nabla \mathbf{u}]+\boldsymbol{\theta} \mathbf{M}) \mathbf{n}=\mathbf{0} \quad \text { on } \quad \partial B_{2} \times(0, \infty), \\
\boldsymbol{\theta}=0 \quad \text { on } \quad \partial B_{3} \times(0, \infty), & \mathbf{n} \cdot \mathbf{K} \nabla \boldsymbol{\theta}+Q \boldsymbol{\theta}=0 \quad \text { on } \partial B_{4} \times(0, \infty),
\end{array}
$$

and the initial conditions

$$
\mathbf{u}(\mathbf{x}, 0)=\mathbf{u}^{0}(\mathbf{x}), \quad \dot{\mathbf{u}}(\mathbf{x}, 0)=\dot{\mathbf{u}}^{0}(\mathbf{x}), \quad \theta(\mathbf{x}, 0)=\theta^{0}(\mathbf{x}), \quad \mathbf{x} \in B,
$$

where $\mathbf{u}^{0}, \dot{\mathbf{u}}^{0}$, and $\theta^{0}$ are prescribed functions in $\mathbf{W}_{1}(B), \mathbf{W}_{0}(B)$, and $W_{0}(B)$, respectively. Here $W_{m}(B)$ represents the familiar Sobolev space [12], and $\mathbf{W}_{m}(B)=\left[W_{m}(B)\right]^{3}$.

In thermodynamic terms, the second relation in (12) states that the part $\partial B_{3}$ of the boundary is kept at a constant temperature $\theta_{0}$, while the rest $\partial B_{4}$ is radiating into a surrounding medium at temperature $\theta_{0}$. The above initial boundary value problem was considered by Brun [13], who proves the uniqueness of solution. 
If meas $\partial B_{1}=0$, then there exists a family of rigid motions and null temperature deviation which satisfy Eqs. (10) and (11) and the boundary conditions (12). From this, we decompose the initial data $\mathbf{u}^{0}$ and $\dot{\mathbf{u}}^{0}$ as follows:

$$
\mathbf{u}^{0}=\mathbf{u}_{*}^{0}+\mathbf{U}^{0}, \quad \dot{\mathbf{u}}^{0}=\dot{\mathbf{u}}_{*}^{0}+\dot{\mathbf{U}}^{0},
$$

where $\mathbf{u}_{*}^{0}$ and $\dot{\mathbf{u}}_{*}^{0}$ are rigid displacements determined so that $\mathbf{U}^{0}$ and $\dot{\mathbf{U}}^{0}$ satisfy the normalization restrictions

$$
\begin{array}{ll}
\int_{B} \rho \mathbf{U}^{0} d V=\mathbf{0}, & \int_{B} \rho \mathbf{x} \wedge \mathbf{U}^{0} d V=\mathbf{0}, \\
\int_{B} \rho \dot{\mathbf{U}}^{0} d V=\mathbf{0}, & \int_{B} \rho \mathbf{x} \wedge \dot{\mathbf{U}}^{0} d V=\mathbf{0} .
\end{array}
$$

Let us introduce the following notations:

$$
\begin{aligned}
& \hat{\mathbf{C}}^{1}(B) \equiv\left\{\mathbf{v} \in\left[C^{1}(\bar{B})\right]^{3}: \mathbf{v}=\mathbf{0} \text { on } \overline{\partial B_{1}} \text { and if meas } \partial B_{1}=0,\right. \\
& \text { then } \left.\int_{B} \rho \mathbf{v} d V=\mathbf{0}, \int_{B} \rho \mathbf{x} \wedge \mathbf{v} d V=\mathbf{0}\right\} ; \\
& \hat{C}^{1}(B) \equiv\left\{\chi \in C^{1}(\bar{B}): \chi=0 \text { on } \overline{\partial B_{3}} \text { and if meas } \partial B_{3}=0\right. \text {, } \\
& \text { then } \mathbf{n} \cdot \mathbf{K} \nabla \chi+Q \chi=0 \text { on } \partial B\} ; \\
& \hat{\mathbf{W}}_{1}(B) \equiv \text { the completion of } \hat{\mathbf{C}}^{1}(B) \text { by means of }\|\cdot\| \mathbf{w}_{1}(B) \text {, } \\
& \hat{W}_{1}(B) \equiv \text { the completion of } \hat{C}^{1}(B) \text { by means of }\|\cdot\| \mathbf{w}_{1}(B) \text {. }
\end{aligned}
$$

In these relations $C^{1}(\bar{B})$ represents the set of scalar functions which are continuous and continuously differentiable on $\bar{B}$.

Let $\mathbf{u}, \mathbf{v} \in \hat{\mathbf{C}}^{1}(B)$ and $\theta, \chi \in \hat{C}^{1}(B)$. We set

$$
\Phi(\mathbf{u}, \mathbf{v}) \equiv \int_{B} \nabla \mathbf{u} \cdot \mathbf{C}[\nabla \mathbf{v}] d V, \quad \Omega(\theta, \chi) \equiv \int_{B} \frac{1}{\theta_{0}} \nabla \theta \cdot \mathbf{K} \nabla \chi d V .
$$

Obviously, $\Phi$ and $\Omega$ may be extended by continuity onto $\hat{\mathbf{W}}_{1}(B) \times \hat{\mathbf{W}}_{1}(B)$ and $\hat{W}_{1}(B) \times$ $\hat{W}_{1}(B)$, respectively.

In order to obtain our results, we make the following further assumptions:

(a) The density and specific heat satisfy the conditions

$$
\begin{aligned}
& 0<\rho_{1} \leqslant \underset{B}{\operatorname{ess.inf}} \rho(\mathbf{x}) \leqslant \underset{B}{\operatorname{ess.sup}} \rho(\mathbf{x}) \leqslant \rho_{2}, \\
& 0<c_{1} \leqslant \underset{B}{\operatorname{ess} . i n f} c(\mathbf{x}) \leqslant \text { ess.sup } c(\mathbf{x}) \leqslant c_{2} .
\end{aligned}
$$

(b) There exist positive constants $m$ and $k$ such that

$$
\begin{gathered}
\Phi(\mathbf{v}, \mathbf{v}) \geqslant m \int_{B}(\operatorname{sym} \nabla \mathbf{v}) \cdot(\operatorname{sym} \nabla \mathbf{v}) d V, \quad \text { for all } \mathbf{v} \in \hat{\mathbf{C}}^{1}(B), \\
\Omega(\chi, \chi) \geqslant k \int_{B} \nabla \chi \cdot \nabla \chi d V, \quad \text { for all } \chi \in \hat{C}^{1}(B) .
\end{gathered}
$$


REMARK 2.1. The assumptions above are in agreement with physical experience. The mechanical interpretation of (16) is obvious. Estimate (17) may be interpreted with the help of the theory of elastic stability [14]. Finally, estimate (18) is related to the defining property of a definite elastic heat conductor and is in accordance with the consequence (9) of the heat conduction inequality.

REMARK 2.2. We note that the hypothesis (17) ensures that the following Korn's inequality [15] holds, for all $\mathbf{v} \in \hat{\mathbf{W}}_{1}(B)$ :

$$
\Phi(\mathbf{v}, \mathbf{v}) \geqslant m_{1} \int_{B}(\mathbf{v} \cdot \mathbf{v}+\nabla \mathbf{v} \cdot \nabla \mathbf{v}) d V, \quad m_{1}=\text { const. }>0 .
$$

Moreover, under hypothesis (18), for all $\chi \in \hat{W}_{1}(B)$, the following Poincaré inequality holds:

$$
\Omega(\chi, \chi)+\int_{\partial B_{4}} \frac{1}{\theta_{0}} Q \chi^{2} d A \geqslant k_{1} \int_{B} \chi^{2} d V, \quad k_{1}=\text { const. }>0 .
$$

REMARK 2.3. Under assumptions similar to the above, Dafermos [16] has demonstrated the existence, regularity, and asymptotic stability of the solutions to the initial boundary value problems defined by relations (10) to (13). Therefore, in what follows it is assumed that a twice continuously differentiable solution $(\mathbf{u}, \theta)(\mathbf{x}, t)$ exists satisfying Eqs. (10) and (11) and conditions (12) and (13).

If meas $\partial B_{1}=0$, then we shall find it a convenient practice to decompose the solution $(\mathbf{u}, \theta)$ in the form

$$
(\mathbf{u}, \theta)=\left(\mathbf{u}_{*}^{0}+t \dot{\mathbf{u}}_{*}^{0}+\mathbf{v}, \chi\right),
$$

where $(\mathbf{v}, \chi) \in \hat{\mathbf{W}}_{1}(B) \times \hat{W}_{1}(B)$ represents the solution of (10)-(12) with the initial conditions

$$
\mathbf{v}(\mathbf{x}, 0)=\mathbf{U}^{0}(\mathbf{x}), \quad \dot{\mathbf{v}}(\mathbf{x}, 0)=\dot{\mathbf{U}}^{0}(\mathbf{x}), \quad \chi(\mathbf{x}, 0)=\theta^{0}(\mathbf{x}), \quad \mathbf{x} \in B .
$$

3. Some evolutionary identities. In order to establish our principal results, we shall need some preliminary integral identities. The first theorem presents a well-known conservation law of energy in linear thermoelasticity.

Theorem 3.1. Suppose that $\mathbf{u}^{0} \in \mathbf{W}_{1}(B), \dot{\mathbf{u}}^{0} \in W_{0}(B), \theta^{0} \in W_{0}(B)$ and that $\mathbf{C}, \mathbf{M}$, and $\mathbf{K}$ satisfy the symmetry relations (6), (7), and (8). Let $(\mathbf{u}, \theta)$ be a solution of the initial boundary value problem defined by (10) to (13). Then the following energy conservation law holds:

$$
\begin{aligned}
\mathscr{E}(t) & \equiv \frac{1}{2} \int_{B}\left\{\rho \dot{\mathbf{u}}(t) \cdot \dot{\mathbf{u}}(t)+\nabla \mathbf{u}(t) \cdot \mathbf{C}[\nabla \mathbf{u}(t)]+\frac{c}{\theta_{0}} \theta^{2}(t)\right\} d V \\
& +\int_{0}^{t} \int_{B} \frac{1}{\theta_{0}} \nabla \theta(s) \cdot \mathbf{K} \nabla \theta(s) d V d s+\int_{0}^{t} \int_{\partial B_{4}} \frac{1}{\theta_{0}} Q \theta^{2}(s) d A d s=\mathscr{E}(0), t \in(0, \infty),
\end{aligned}
$$

where we have omitted to mention the explicit dependence of functions on their spatial argument. 
Proof. We multiply Eq. (10) by $\dot{\mathbf{u}}$ and (11) by $\left(1 / \theta_{0}\right) \theta$ and add the results. Then we integrate the result over $B \times(0, t), t \in(0, \infty)$, and use integration by parts. Further, we make use of conditions (6), (7), (8), (12), and (13) in order to obtain (22). The proof is complete.

We shall find it convenient to introduce the following notations associated with a solution $(\mathbf{u}, \boldsymbol{\theta})$ of $(10)$ to (13),

$$
\begin{aligned}
\mathscr{K}(t) & \equiv \frac{1}{2} \int_{B} \rho \dot{\mathbf{u}}(t) \cdot \dot{\mathbf{u}}(t) d V, \\
\mathscr{S}(t) & \equiv \frac{1}{2} \int_{B} \nabla \mathbf{u}(t) \cdot \mathbf{C}[\nabla \mathbf{u}(t)] d V, \\
\mathscr{T}(t) & \equiv \frac{1}{2} \int_{B} \frac{c}{\theta_{0}} \theta^{2}(t) d V, \\
\Gamma(t) & \equiv \int_{0}^{t} \int_{B} \frac{1}{\theta_{0}} \nabla \theta(s) \cdot \mathbf{K} \nabla \theta(s) d V d s, \\
\Lambda(t) & \equiv \int_{0}^{t} \int_{\partial B_{4}} \frac{1}{\theta_{0}} Q \theta^{2}(s) d A d s .
\end{aligned}
$$

Thus, the energy conservation law (22) can be written in the form

$$
\mathscr{E}(t) \equiv \mathscr{K}(t)+\mathscr{S}(t)+\mathscr{T}(t)+\Gamma(t)+\Lambda(t)=\mathscr{E}(0) .
$$

REMARK 3.1. We notice that $\mathscr{K}$ represents the global kinetic energy of $B, \mathscr{S}$ represents the strain energy of $B, \mathscr{T}$ is the thermal energy of $B, \Gamma$ represents the thermal entropy product on $(0, t)$ or the dissipation energy in the body due to the thermal irreversibilities, and $\Lambda$ represents the total heat supplied into $B$ on $(0, t)$ by conduction across $\partial B_{4}$. In the next section, we shall establish the asymptotic partition of energy $\mathscr{E}(t)$ in terms of the Cesàro means of the energies defined by (23).

We now establish some Lagrange-Brun type identities. Thus, we have

TheOREM 3.2. Suppose that the conditions of Theorem 3.1 hold. Let $(\mathbf{u}, \theta)$ be a solution of the initial boundary value problem defined by (10) to (13). Then the following identity holds:

$$
\begin{aligned}
2 \int_{B} \rho \mathbf{u}(t) \cdot \dot{\mathbf{u}}(t) d V & +\int_{B} \frac{1}{\theta_{0}}\left(\int_{0}^{t} \nabla \theta(z) d z\right) \cdot \mathbf{K}\left(\int_{0}^{t} \nabla \theta(z) d z\right) d V \\
& +\int_{\partial B_{4}} \frac{1}{\theta_{0}} Q\left(\int_{0}^{t} \theta(z) d z\right)^{2} d A \\
& =2 \int_{0}^{t} \int_{B}\left\{\rho \dot{\mathbf{u}}(s) \cdot \dot{\mathbf{u}}(s)-\nabla \mathbf{u}(s) \cdot \mathbf{C}[\nabla \mathbf{u}(s)]-\frac{c}{\theta_{0}} \theta^{2}(s)\right\} d V d s \\
& +2 \int_{B} \rho \mathbf{u}^{0} \cdot \dot{\mathbf{u}}^{0} d V+2 \int_{0}^{t} \int_{B} \eta_{0} \theta(s) d V d s, \quad t \in(0, \infty),
\end{aligned}
$$

where

$$
\eta_{0}(\mathbf{x}) \equiv-\mathbf{M}(\mathbf{x}) \cdot \nabla \mathbf{u}^{0}(\mathbf{x})+\frac{1}{\theta_{0}} c(\mathbf{x}) \theta^{0}(\mathbf{x})
$$


Proof. We start from the following identity:

$$
\frac{d}{d s}[\rho \mathbf{u}(s) \cdot \dot{\mathbf{u}}(s)]=\rho \dot{\mathbf{u}}(s) \cdot \dot{\mathbf{u}}(s)+\rho \mathbf{u}(s) \cdot \ddot{\mathbf{u}}(s) .
$$

By an integration over $B \times(0, t)$ and using conditions (13), we get

$$
\int_{B} \rho \mathbf{u}(t) \cdot \dot{\mathbf{u}}(t) d V=\int_{B} \rho \mathbf{u}^{0} \cdot \dot{\mathbf{u}}^{0} d V+\int_{0}^{t} \int_{B}[\rho \dot{\mathbf{u}}(s) \cdot \dot{\mathbf{u}}(s)+\rho \mathbf{u}(s) \cdot \ddot{\mathbf{u}}(s)] d V d s .(27)
$$

We next eliminate the inertial term on the right-hand side of (27) by means of (10). Then we integrate by parts with respect to the spatial variable and use (6), (7), and (12) to obtain

$$
\begin{aligned}
\int_{B} \rho \mathbf{u}(t) \cdot \dot{\mathbf{u}}(t) d V= & \int_{B} \rho \mathbf{u}^{0} \cdot \dot{\mathbf{u}}^{0} d V+\int_{0}^{t} \int_{B}\{\rho \dot{\mathbf{u}}(s) \cdot \dot{\mathbf{u}}(s)-\nabla \mathbf{u}(s) \cdot \mathbf{C}[\nabla \mathbf{u}(s)] \\
& -\mathbf{M} \cdot \nabla \mathbf{u}(s) \theta(s)\} d V d s .
\end{aligned}
$$

On the other hand, by integration with respect to the time variable, from (11) and (13), we deduce

$$
\mathbf{M} \cdot \nabla \mathbf{u}(t)=\frac{c}{\theta_{0}} \theta(t)-\frac{1}{\theta_{0}} \operatorname{div}\left(\mathbf{K} \int_{0}^{t} \nabla \theta(z) d z\right)-\eta_{0},
$$

where $\eta_{0}(\mathbf{x})$ is defined by (26). Further, we use Eq. (29) in (28). By integration by parts followed by the use of (12), we obtain

$$
\begin{aligned}
\int_{B} \rho \mathbf{u}(t) \cdot \dot{\mathbf{u}}(t) d V & +\int_{0}^{t} \int_{B} \frac{1}{\theta_{0}} \nabla \theta(s) \cdot \mathbf{K}\left(\int_{0}^{s} \nabla \theta(z) d z\right) d V d s \\
& +\int_{0}^{t} \int_{\partial B_{4}} \frac{1}{\theta_{0}} Q \theta(s) \int_{0}^{s} \theta(z) d z d A d s \\
= & \int_{0}^{t} \int_{B}\left\{\rho \dot{\mathbf{u}}(s) \cdot \dot{\mathbf{u}}(s)-\nabla \mathbf{u}(s) \cdot \mathbf{C}[\nabla \mathbf{u}(s)]-\frac{c}{\theta_{0}} \theta^{2}(s)\right\} d V d s \\
& +\int_{B} \rho \mathbf{u}^{0} \cdot \dot{\mathbf{u}}^{0} d V+\int_{0}^{t} \int_{B} \eta_{0} \theta(s) d V d s, \quad t \in(0, \infty) .
\end{aligned}
$$

It follows from (30) that (25) holds. This completes the proof.

Theorem 3.3. Suppose that the conditions of Theorem 3.1 hold. Let $(\mathbf{u}, \theta)$ be a solution of the initial boundary value problem defined by (10) to (13). Then the following identity holds:

$$
\begin{aligned}
2 \int_{B} \rho \mathbf{u}(t) \cdot \dot{\mathbf{u}}(t) d V+\int_{B} \frac{1}{\theta_{0}}\left(\int_{0}^{t} \nabla \theta(z) d z\right) \cdot \mathbf{K}\left(\int_{0}^{t} \nabla \theta(z) d z\right) d V \\
+\int_{\partial B_{4}} \frac{1}{\theta_{0}} Q\left(\int_{0}^{t} \theta(z) d z\right)^{2} d A \\
=\int_{B}\left\{\rho \dot{\mathbf{u}}^{0} \cdot \mathbf{u}(2 t)+\rho \mathbf{u}^{0} \cdot \dot{\mathbf{u}}(2 t)\right\} d V+\int_{0}^{t} \int_{B} \eta_{0} \theta(s) d V d s-\int_{t}^{2 t} \int_{B} \eta_{0} \theta(s) d V d s \\
t \in(0, \infty)
\end{aligned}
$$


Proof. Let $\mathbf{f}(\cdot, s)$ and $\mathbf{g}(\cdot, s)$ be vector fields assumed to be twice continuously differentiable with respect to the time variable $s$. Obviously, we find that

$$
\frac{d}{d s}\{\rho[\mathbf{f}(s) \cdot \dot{\mathbf{g}}(s)-\dot{\mathbf{f}}(s) \cdot \mathbf{g}(s)]\}=\rho[\mathbf{f}(s) \cdot \ddot{\mathbf{g}}(s)-\ddot{\mathbf{f}}(s) \cdot \mathbf{g}(s)] .
$$

By integration of this identity over $B \times(0, t), t \in(0, \infty)$, we deduce

$$
\begin{aligned}
\int_{B} \rho[\mathbf{f}(t) \cdot \dot{\mathbf{g}}(t)-\dot{\mathbf{f}}(t) \cdot \mathbf{g}(t)] d V= & \int_{0}^{t} \int_{B} \rho[\mathbf{f}(s) \cdot \ddot{\mathbf{g}}(s)-\ddot{\mathbf{f}}(s) \cdot \mathbf{g}(s)] d V d s \\
& +\int_{B} \rho[\mathbf{f}(0) \cdot \dot{\mathbf{g}}(0)-\dot{\mathbf{f}}(0) \cdot \mathbf{g}(0)] d V .
\end{aligned}
$$

We now set

$$
\mathbf{f}(\mathbf{x}, \tau)=\mathbf{u}(\mathbf{x}, \tau), \quad \mathbf{g}(\mathbf{x}, \tau)=\mathbf{u}(\mathbf{x}, 2 t-\tau), \quad \tau \in[0,2 t], t \in(0, \infty),
$$

so that (32) becomes

$$
\begin{aligned}
2 \int_{B} \rho \mathbf{u}(t) \cdot \dot{\mathbf{u}}(t) d V=\int_{0}^{t} \int_{B} \rho[\mathbf{u}(2 t-s) \cdot \ddot{\mathbf{u}}(s)-\mathbf{u}(s) \cdot \ddot{\mathbf{u}}(2 t-s)] d V d s & \\
& +\int_{B} \rho\left[\dot{\mathbf{u}}^{0} \cdot \mathbf{u}(2 t)+\mathbf{u}^{0} \cdot \dot{\mathbf{u}}(2 t)\right] d V, \quad t \in(0, \infty) .
\end{aligned}
$$

Substitution of the inertial terms on the right-hand side of (33) and application of the divergence theorem, followed by use of relations (6), (7), and (12), lead to

$$
\begin{aligned}
2 \int_{B} \rho \mathbf{u}(t) \cdot \dot{\mathbf{u}}(t) d V=\int_{0}^{t} \int_{B}[ & \theta(2 t-s) \mathbf{M} \cdot \nabla \mathbf{u}(s)-\theta(s) \mathbf{M} \cdot \nabla \mathbf{u}(2 t-s)] d V d s \\
& +\int_{B} \rho\left[\dot{\mathbf{u}}^{0} \cdot \mathbf{u}(2 t)+\mathbf{u}^{0} \cdot \dot{\mathbf{u}}(2 t)\right] d V, \quad t \in(0, \infty) .
\end{aligned}
$$

Further, we use Eq. (29) in order to obtain

$$
\begin{aligned}
\int_{B}[\theta(2 t-s) \mathbf{M} & \cdot \nabla \mathbf{u}(s)-\theta(s) \mathbf{M} \cdot \nabla \mathbf{u}(2 t-s)] d V \\
= & \int_{B} \frac{1}{\theta_{0}}\left\{\nabla \theta(2 t-s) \cdot \mathbf{K}\left(\int_{0}^{s} \nabla \theta(z) d z\right)\right. \\
& \left.-\nabla \theta(s) \cdot \mathbf{K}\left(\int_{0}^{2 t-s} \nabla \theta(z) d z\right)\right\} d V+\int_{B} \eta_{0}[\theta(s)-\theta(2 t-s)] d V \\
& +\int_{\partial B_{4}} \frac{1}{\theta_{0}} Q\left[\theta(2 t-s) \int_{0}^{s} \theta(z) d z-\theta(s) \int_{0}^{2 t-s} \theta(z) d z\right] d A, \\
&
\end{aligned}
$$


Therefore, the identity (34) takes the form

$$
\begin{aligned}
& 2 \int_{B} \rho \mathbf{u}(t) \cdot \dot{\mathbf{u}}(t) d V \\
&= \int_{0}^{t} \int_{B} \frac{1}{\theta_{0}}\left\{\nabla \theta(2 t-s) \cdot \mathbf{K}\left(\int_{0}^{s} \nabla \theta(z) d z\right)-\nabla \theta(s) \cdot \mathbf{K}\left(\int_{0}^{2 t-s} \nabla \theta(z) d z\right)\right\} d V d s \\
&+\int_{0}^{t} \int_{\partial B_{4}} \frac{1}{\theta_{0}} Q\left[\theta(2 t-s) \int_{0}^{s} \theta(z) d z-\theta(s) \int_{0}^{2 t-s} \theta(z) d z\right] d A d s \\
&+\int_{B} \rho\left[\dot{\mathbf{u}}^{0} \cdot \mathbf{u}(2 t)+\mathbf{u}^{0} \cdot \dot{\mathbf{u}}(2 t)\right] d V+\int_{0}^{t} \int_{B} \eta_{0}[\theta(s)-\theta(2 t-s)] d V d s \\
& \quad t \in(0, \infty)
\end{aligned}
$$

Since

$$
\begin{gathered}
\int_{0}^{t} \int_{B} \frac{1}{\theta_{0}} \frac{d}{d s}\left(\int_{0}^{s} \nabla \theta(z) d z\right) \cdot \mathbf{K}\left(\int_{0}^{2 t-s} \nabla \theta(z) d z\right) d V d s \\
=\int_{B} \frac{1}{\theta_{0}}\left(\int_{0}^{t} \nabla \theta(z) d z\right) \cdot \mathbf{K}\left(\int_{0}^{t} \nabla \theta(z) d z\right) d V \\
\quad+\int_{0}^{t} \int_{B} \frac{1}{\theta_{0}} \nabla \theta(2 t-s) \cdot \mathbf{K}\left(\int_{0}^{s} \nabla \theta(z) d z\right) d V d s, \\
\int_{0}^{t} \int_{\partial B_{4}} \frac{1}{\theta_{0}} Q \frac{d}{d s}\left(\int_{0}^{s} \theta(z) d z\right)\left(\int_{0}^{2 t-s} \theta(z) d z\right) d A d s \\
=\int_{\partial B_{4}} \frac{1}{\theta_{0}} Q\left(\int_{0}^{t} \theta(z) d z\right)^{2} d A+\int_{0}^{t} \int_{\partial B_{4}} \frac{1}{\theta_{0}} Q \theta(2 t-s) \int_{0}^{s} \theta(z) d z d A d s,
\end{gathered}
$$

with the aid of (8), from (35) we get (31). Thus, the proof is complete.

An immediate consequence of Theorem 3.3 is the following uniqueness result.

Corollary 3.1. Suppose that $\mathbf{C}, \mathbf{M}$, and $\mathbf{K}$ satisfy the symmetry relations (6), (7), and (8) and that $\rho$ is strictly positive and the thermal conductivity tensor $\mathbf{K}$ is positive definite in the sense of relation (18). Let $(\mathbf{u}, \theta)$ be a solution of the initial boundary value problem defined by relations (10) to (12) and by the initial conditions

$$
\mathbf{u}(\mathbf{x}, 0)=\mathbf{0}, \quad \dot{\mathbf{u}}(\mathbf{x}, 0)=\mathbf{0}, \quad \theta(\mathbf{x}, 0)=0 \quad \text { in } B .
$$

Then

$$
\mathbf{u}(\mathbf{x}, t)=\mathbf{0}, \quad \theta(\mathbf{x}, t)=0 \quad \text { for all }(\mathbf{x}, t) \in B \times[0, \infty) .
$$

Proof. Using the initial conditions (36), the identity (31) implies

$$
\begin{aligned}
\int_{B} \rho \mathbf{u}(\tau) \cdot \mathbf{u}(\tau) d V+\int_{0}^{\tau} \int_{B} & \frac{1}{\theta_{0}}\left(\int_{0}^{t} \nabla \theta(z) d z\right) \cdot \mathbf{K}\left(\int_{0}^{t} \nabla \theta(z) d z\right) d V d t \\
& +\int_{0}^{\tau} \int_{\partial B_{4}} \frac{1}{\theta_{0}} Q\left(\int_{0}^{t} \theta(z) d z\right)^{2} d A d t=0, \quad \tau \in(0, \infty) .
\end{aligned}
$$


In view of hypothesis (18), from identity (38) there results

$$
\begin{gathered}
\mathbf{u}(\mathbf{x}, t)=\mathbf{0}, \quad \nabla \theta(\mathbf{x}, t)=\mathbf{0}, \quad(\mathbf{x}, t) \in B \times(0, \infty), \\
\theta(\mathbf{x}, t)=0, \quad(\mathbf{x}, t) \in \partial B_{4} \times(0, \infty) .
\end{gathered}
$$

This proves relation (37) and the proof is complete.

REMARK 3.2. Corollary 3.1 represents a uniqueness result similar to that obtained by Brun [13]. We note that, in contrast to [13], we have not used the energy conservation law.

4. Asymptotic partition of energy in the Cesàro sense. In this section we shall use identities (22), (25), and (31) in conjunction with the hypotheses made in Sec. 2 in order to establish the asymptotic partition of total energy defined by (24). In this aim, we introduce the Cesàro means of various energies defined in (23). Thus, we define

$$
\begin{aligned}
\mathscr{K}_{C}(t) & \equiv \frac{1}{t} \int_{0}^{t} \mathscr{K}(s) d s=\frac{1}{2 t} \int_{0}^{t} \int_{B} \rho \dot{\mathbf{u}}(s) \cdot \dot{\mathbf{u}}(s) d V d s, \\
\mathscr{S}_{C}(t) & \equiv \frac{1}{t} \int_{0}^{t} \mathscr{S}(s) d s=\frac{1}{2 t} \int_{0}^{t} \int_{B} \nabla \mathbf{u}(s) \cdot \mathbf{C}[\nabla \mathbf{u}(s)] d V d s, \\
\mathscr{T}_{C}(t) & \equiv \frac{1}{t} \int_{0}^{t} \mathscr{T}(s) d s=\frac{1}{2 t} \int_{0}^{t} \int_{B} \frac{c}{\theta_{0}} \theta^{2}(s) d V d s, \\
\Gamma_{C}(t) & \equiv \frac{1}{t} \int_{0}^{t} \Gamma(s) d s=\frac{1}{t} \int_{0}^{t} \int_{0}^{s} \int_{B} \frac{1}{\theta_{0}} \nabla \theta(z) \cdot \mathbf{K} \nabla \theta(z) d V d z d s, \\
\Lambda_{C}(t) & \equiv \frac{1}{t} \int_{0}^{t} \Lambda(s) d s=\frac{1}{t} \int_{0}^{t} \int_{0}^{s} \int_{\partial B_{4}} \frac{1}{\theta_{0}} Q \theta^{2}(z) d A d z d s, \quad t \in(0, \infty) .
\end{aligned}
$$

Remark 4.1. The Cesàro mean values of the temperature, heat flux, displacement, strain, and stress have been introduced by Day [17, 18]. It was shown by Day that these mean values can be found by solving an equilibrium boundary value problem which is more tractable than the original dynamic problem.

We have now assembled all the preliminary material needed to derive the asymptotic partition of energy in terms of the mean energies defined by (39).

Theorem 4.1. Suppose that $\mathbf{C}, \mathbf{M}$, and $\mathbf{K}$ satisfy the symmetry relations (6), (7), and (8) and together with the specfic heat $c$ and the density $\rho$ verify the hypotheses (16) to (18). Let $(u, \theta)$ be a solution of the initial boundary value problem defined by (10) to (13). Then for all choices of initial data $\mathbf{u}^{0} \in \mathbf{W}_{1}(B), \dot{\mathbf{u}}^{0} \in \mathbf{W}_{0}(B), \theta^{0} \in W_{0}(B)$,

$$
\lim _{t \rightarrow \infty} \mathscr{T}_{C}(t)=0 .
$$

Moreover, the following assertions hold:

(i) If meas $\partial B_{1} \neq 0$, then

$$
\begin{gathered}
\lim _{t \rightarrow \infty} \mathscr{K}_{C}(t)=\lim _{t \rightarrow \infty} \mathscr{S}_{C}(t), \\
\lim _{t \rightarrow \infty}\left[\Gamma_{C}(t)+\Lambda_{C}(t)\right]=\mathscr{E}(0)-2 \lim _{t \rightarrow \infty} \mathscr{K}_{C}(t)=\mathscr{E}(0)-2 \lim _{t \rightarrow \infty} \mathscr{S}_{C}(t) .
\end{gathered}
$$


(ii) If meas $\partial B_{1}=0$, then

$$
\begin{aligned}
\lim _{t \rightarrow \infty} \mathscr{K}_{C}(t) & =\lim _{t \rightarrow \infty} \mathscr{S}_{C}(t)+\frac{1}{2} \int_{B} \rho \dot{\mathbf{u}}_{*}^{0} \cdot \dot{\mathbf{u}}_{*}^{0} d V \\
\lim _{t \rightarrow \infty}\left[\Gamma_{C}(t)+\Lambda_{C}(t)\right] & =\mathscr{E}(0)-2 \lim _{t \rightarrow \infty} \mathscr{K}_{C}(t)+\frac{1}{2} \int_{B} \rho \dot{\mathbf{u}}_{*}^{0} \cdot \dot{\mathbf{u}}_{*}^{0} d V \\
& =\mathscr{E}(0)-2 \lim _{t \rightarrow \infty} \mathscr{S}_{C}(t)-\frac{1}{2} \int_{B} \rho \dot{\mathbf{u}}_{*}^{0} \cdot \dot{\mathbf{u}}_{*}^{0} d V
\end{aligned}
$$

Proof. We first use the energy conservation law (22) in conjunction with hypotheses (16) to (18) and relations (19) and (20) in order to prove relation (40). Thus, from (18), (20), and (22), we deduce

$$
\begin{aligned}
\int_{0}^{\tau} \int_{B} \theta^{2}(s) d V d s & \leqslant \frac{1}{k_{1}}\left\{\int_{0}^{\tau} \int_{B} \frac{1}{\theta_{0}} \nabla \theta(s) \cdot \mathbf{K} \nabla \theta(s) d V d s+\int_{0}^{\tau} \int_{\partial B_{4}} \frac{1}{\theta_{0}} Q \theta^{2}(s) d A d s\right\} \\
& \leqslant \frac{1}{k_{1}} \mathscr{E}(0), \quad \tau \in(0, \infty)
\end{aligned}
$$

Now, we use hypothesis (16) to obtain

$$
\int_{0}^{\tau} \int_{B} \frac{c}{\theta_{0}} \theta^{2}(s) d V d s \leqslant \frac{c_{2}}{\theta_{0}} \int_{0}^{\tau} \int_{B} \theta^{2}(s) d V d s
$$

so that (45) implies

$$
\mathscr{T}_{C}(\tau) \leqslant \frac{c_{2}}{2 k_{1} \theta_{0}} \cdot \frac{\mathscr{E}(0)}{\tau}
$$

Letting $\tau \rightarrow \infty$ in (46), we deduce relation (40).

Further, we use identities (25) and (31) to obtain

$$
\begin{aligned}
\int_{0}^{t} \int_{B} \rho \dot{\mathbf{u}}(s) \cdot \dot{\mathbf{u}}(s) d V d s-\int_{0}^{t} \int_{B} \nabla \mathbf{u}(s) \cdot \mathbf{C}[\nabla \mathbf{u}(s)] d V d s \\
=\int_{0}^{t} \int_{B} \frac{c}{\theta_{0}} \theta^{2}(s) d V d s-\int_{B} \rho \mathbf{u}^{0} \cdot \dot{\mathbf{u}}^{0} d V+\frac{1}{2} \int_{B} \rho\left[\mathbf{u}^{0} \cdot \dot{\mathbf{u}}(2 t)+\dot{\mathbf{u}}^{0} \cdot \mathbf{u}(2 t)\right] d V \\
\quad-\frac{1}{2} \int_{0}^{t} \int_{B} \eta_{0} \theta(s) d V d s-\frac{1}{2} \int_{t}^{2 t} \int_{B} \eta_{0} \theta(s) d V d s
\end{aligned}
$$

To estimate the right-hand side of the identity (47) we shall use relation (46), the energy conservation law (22), and hypotheses (16) to (18). Thus, we have

$$
\int_{B} \rho \dot{\mathbf{u}}(\tau) \cdot \dot{\mathbf{u}}(\tau) d V \leqslant 2 \mathscr{E}(0), \quad \tau \in(0, \infty) .
$$

Let us first suppose that $\partial B_{1} \neq \varnothing$. Then $\mathbf{u} \in \hat{\mathbf{W}}_{1}(B)$ and from (17), (19), and (22) we get

$$
\int_{B} \rho \mathbf{u}(\tau) \cdot \mathbf{u}(\tau) d V \leqslant \frac{\rho_{2}}{m_{1}} \int_{B} \nabla \mathbf{u}(\tau) \cdot \mathbf{C}[\nabla \mathbf{u}(\tau)] d V \leqslant \frac{2 \rho_{2}}{m_{1}} \mathscr{E}(0), \quad \tau \in(0, \infty) .
$$


Now we use the Schwarz and Cauchy inequalities and the estimates (45), (48), and (49), so that, with notations (39), we deduce from (47)

$$
\begin{aligned}
\left|\mathscr{K}_{C}(t)-\mathscr{S}_{C}(t)\right|= & \mid \mathscr{T}_{C}(t)-\frac{1}{2 t} \int_{B} \rho \mathbf{u}^{0} \cdot \dot{\mathbf{u}}^{0} d V+\frac{1}{4 t} \int_{B} \rho\left[\mathbf{u}^{0} \cdot \dot{\mathbf{u}}(2 t)+\dot{\mathbf{u}}^{0} \cdot \mathbf{u}(2 t)\right] d V \\
& -\frac{1}{4 t} \int_{0}^{t} \int_{B} \eta_{0} \theta(s) d V d s-\frac{1}{4 t} \int_{t}^{2 t} \int_{B} \eta_{0} \theta(s) d V d s \mid \\
\leqslant & \mathscr{T}_{C}(t)+\frac{1}{4 t} \int_{B} \rho\left(\mathbf{u}^{0} \cdot \mathbf{u}^{0}+\dot{\mathbf{u}}^{0} \cdot \dot{\mathbf{u}}^{0}\right) d V \\
& +\frac{1}{8 t} \int_{B} \rho\left[\mathbf{u}^{0} \cdot \mathbf{u}^{0}+\dot{\mathbf{u}}^{0} \cdot \dot{\mathbf{u}}^{0}+\dot{\mathbf{u}}(2 t) \cdot \dot{\mathbf{u}}(2 t)\right. \\
& +\mathbf{u}(2 t) \cdot \mathbf{u}(2 t)] d V+\frac{1}{4 t}\left(\int_{0}^{t} \int_{B} \eta_{0}^{2} d V d s\right)^{1 / 2}\left(\int_{0}^{t} \int_{B} \theta^{2}(s) d V d s\right)^{1 / 2} \\
& +\frac{1}{4 t}\left(\int_{t}^{2 t} \int_{B} \eta_{0}^{2} d V d s\right)^{1 / 2}\left(\int_{t}^{2 t} \int_{B} \theta^{2}(s) d V d s\right)^{1 / 2} \\
\leqslant & \mathscr{T}_{C}(t)+\frac{3}{8 t} \int_{B} \rho\left(\mathbf{u}^{0} \cdot \mathbf{u}^{0}+\dot{\mathbf{u}}^{0} \cdot \dot{\mathbf{u}}^{0}\right) d V+\frac{1}{4 t} \mathscr{E}(0)\left(1+\frac{\rho_{2}}{m_{1}}\right) \\
& +\frac{1}{2} t^{-1 / 2}\left(\int_{B} \eta_{0}^{2} d V\right)^{1 / 2}\left(\frac{1}{k_{1}} \mathscr{E}(0)\right)^{1 / 2} \cdot
\end{aligned}
$$

We make $t \rightarrow \infty$ in (50) and we take into account relation (40) so that we obtain relation (41). Relation (42) is obtained from (22) by taking the Cesàro means and using relations (40) and (41).

Let us now consider the case meas $\partial B_{1}=0$. If we use the decomposition (21), we get

$$
\begin{aligned}
\frac{1}{4 t} \int_{B} \rho \dot{\mathbf{u}}^{0} \cdot \mathbf{u}(2 t) d V & =\frac{1}{4 t} \int_{B} \rho\left(\dot{\mathbf{u}}_{*}^{0}+\dot{\mathbf{U}}^{0}\right) \cdot\left[\mathbf{u}_{*}^{0}+2 \dot{\mathbf{u}}_{*}^{0} t+\mathbf{v}(2 t)\right] d V \\
& =\frac{1}{4 t} \int_{B} \rho \dot{\mathbf{u}}_{*}^{0} \cdot \mathbf{u}_{*}^{0} d V+\frac{1}{2} \int_{B} \rho \dot{\mathbf{u}}_{*}^{0} \cdot \dot{\mathbf{u}}_{*}^{0} d V+\frac{1}{4 t} \int_{B} \rho \dot{\mathbf{U}}^{0} \cdot \mathbf{v}(2 t) d V .
\end{aligned}
$$

In deducing relation (51) we have used relations (14), (15), and the fact that $\mathbf{v} \in \hat{\mathbf{W}}_{1}(B)$. In this case, from identity (47) we deduce

$$
\begin{aligned}
\mathscr{K}_{C}(t)- & \mathscr{S}_{C}(t)-\frac{1}{2} \int_{B} \rho \dot{\mathbf{u}}_{*}^{0} \cdot \dot{\mathbf{u}}_{*}^{0} d V \\
= & \mathscr{T}_{C}(t)-\frac{1}{2 t} \int_{B} \rho \dot{\mathbf{u}}^{0} \cdot \mathbf{u}^{0} d V+\frac{1}{4 t} \int_{B} \rho \mathbf{u}^{0} \cdot \dot{\mathbf{u}}(2 t) d V+\frac{1}{4 t} \int_{B} \rho \dot{\mathbf{u}}_{*}^{0} \cdot \mathbf{u}_{*}^{0} d V \\
& +\frac{1}{4 t} \int_{B} \rho \dot{\mathbf{U}}^{0} \cdot \mathbf{v}(2 t) d V-\frac{1}{4 t} \int_{0}^{t} \int_{B} \eta_{0} \theta(s) d V d s-\frac{1}{4 t} \int_{t}^{2 t} \int_{B} \eta_{0} \theta(s) d V d s .
\end{aligned}
$$


Further, we use estimates (45) and (48) in conjunction with

$$
\begin{aligned}
\int_{B} \rho \mathbf{v}(\tau) \cdot \mathbf{v}(\tau) d V & \leqslant \frac{\rho_{2}}{m_{1}} \int_{B} \nabla \mathbf{v}(\tau) \cdot \mathbf{C}[\nabla \mathbf{v}(\tau)] d V \\
& =\frac{\rho_{2}}{m_{1}} \int_{B} \nabla \mathbf{u}(\tau) \cdot \mathbf{C}[\nabla \mathbf{u}(\tau)] d V \leqslant \frac{2 \rho_{2}}{m_{1}} \mathscr{E}(0), \quad \tau \in(0, \infty),
\end{aligned}
$$

to obtain from (52) an estimate similar to (50). This leads immediately to (43) and (44). Thus, the theorem is proved.

REMARK 4.2. Relations (41) and (43) (restricted to the class of initial data for which $\dot{\mathbf{u}}_{*}^{0}=\mathbf{0}$ ) prove the asymptotic equipartition of the mean kinetic and strain energies.

REMARK 4.3. It is worth noting that the coupling factor $\mathbf{M}$ does not appear explicitly in our relations expressing the partition of energy.

Let us consider a fictive thermoelastic continuum for which $\mathbf{M}=\mathbf{0}$. Then Eqs. (10) and (11) become uncoupled. In this case we obtain more precise information about the asymptotic behavior of the mean energies. Thus, the analysis proves that relation (40) continues to hold true, while relations (41) to (44) become

$$
\lim _{t \rightarrow \infty}\left[\Gamma_{C}(t)+\Lambda_{C}(t)\right]=\mathscr{T}(0) .
$$

(i) If meas $\partial B_{1} \neq 0$, then

$$
\lim _{t \rightarrow \infty} \mathscr{K}_{C}(t)=\lim _{t \rightarrow \infty} \mathscr{S}_{C}(t)=\frac{1}{2}[\mathscr{K}(0)+\mathscr{S}(0)] .
$$

(ii) If meas $\partial B_{1}=0$, then

$$
\begin{aligned}
& \lim _{t \rightarrow \infty} \mathscr{K}_{C}(t)=\frac{1}{2}[\mathscr{K}(0)+\mathscr{S}(0)]+\frac{1}{4} \int_{B} \rho \dot{\mathbf{u}}_{*}^{0} \cdot \dot{\mathbf{u}}_{*}^{0} d V, \\
& \lim _{t \rightarrow \infty} \mathscr{S}_{C}(t)=\frac{1}{2}[\mathscr{K}(0)+\mathscr{S}(0)]-\frac{1}{4} \int_{B} \rho \dot{\mathbf{u}}_{*}^{0} \cdot \dot{\mathbf{u}}_{*}^{0} d V .
\end{aligned}
$$

It will be recognized that relations (54) and (55) describe the partition of energy in linear elastodynamics, while relations (40) and (53) describe the asymptotic behavior of the Cesàro means of $\mathscr{T}(t), \Gamma(t)$, and $\Lambda(t)$ associated with the parabolic equation of heat conduction.

\section{REFERENCES}

[1] P. D. Lax and R. S. Phillips, Scattering theory, Academic Press, New York, 1967

[2] A. R. Brodsky, On the asymptotic hehavior of solutions of the wave equation, Proc. Amer. Math. Soc. 18, 207-208 (1967)

[3] J. A. Goldstein, An asymptotic property of solutions of wave equations, Proc. Amer. Math. Soc. 23, 359-363 (1969)

[4] J. A. Goldstein, An asymptotic property of solutions of wave equations, II, J. Math. Anal. Appl. 32, 392-399 (1970)

[5] R. J. Duffin, Equipartition of energy in wave motion, J. Math. Anal. Appl. 32, 386-391 (1970)

[6] H. A. Levine, An equipartition of energy theorem for weak solutions of evolutionary equations in Hilbert space: the Lagrange identity method, J. Differential Equations 24, 197-210 (1977)

[7] W. A. Day, Means and autocorrelations in elastodynamics, Arch. Rat. Mech. Anal. 73, 243-256 (1980)

[8] H. A. Levine, On a theorem of Knops and Payne in dynamical linear thermoelasticity, Arch. Rat. Mech. Anal. 38, 290-319 (1970) 
[9] G. Dassios and M. Grillakis, Dissipation rates and partition of energv in thermoelasticity, Arch. Rat. Mech. Anal. 87, 49-91 (1984)

[10] G. Fichera, Existence theorems in elasticity, in Handbuch der physik, vol. VIa/2, ed. C. Truesdell, Springer, Berlin, 1972

[11] D. E. Carlson, Linear thermoelasticity, in Handbuch der physik, vol. VIa/2, ed. C. Truesdell, Springer, Berlin, 1972

[12] R. A. Adams, Sobolev spaces, Academic Press, New York, 1975

[13] L. Brun, Méthodes énergétiques dans les systèmes évolutifs linéaires, J. Mécanique 8, 125-192 (1969)

[14] R. J. Knops and E. W. Wilkes, Theory of elastic stability, in Handbuch der physik, vol. VIa/3, ed. C. Truesdell, Springer, Berlin, 1973

[15] I. Hlaváček and J. Nečas, On inequalities of Korn's type, Arch. Rat. Mech. Anal. 36, 305-334 (1970)

[16] C. M. Dafermos, On the existence and the asymptotic stability of solutions to the equations of linear thermoelasticity, Arch. Rat. Mech. Anal. 29, 241-271 (1968)

[17] W. A. Day, Cesàro means and recurrence in dynamic thermoelasticity, Mathematika 28, 211-230 (1981)

[18] W. A. Day, Mean and recurrence properties of the temperature in dynamic thermoelasticity, J. Elasticity 13, 225-230 (1983) 